\title{
Multiplexing Biochemical Signals
}

\author{
Wiet de Ronde, Filipe Tostevin, and Pieter Rein ten Wolde \\ FOM Institute for Atomic and Molecular Physics (AMOLF), Science Park 104, 1098 XG Amsterdam, The Netherlands
}

(Received 2 August 2010; published 19 July 2011)

\begin{abstract}
In this Letter we show that living cells can multiplex biochemical signals, i.e., transmit multiple signals through the same signaling pathway simultaneously, and yet respond to them very specifically. We demonstrate how two binary input signals can be encoded in the concentration of a common signaling protein, which is then decoded such that each of the two output signals provides reliable information about one corresponding input. Under biologically relevant conditions the network can reach the maximum amount of information that can be transmitted, which is 2 bits.
\end{abstract}

DOI: 10.1103/PhysRevLett.107.048101

PACS numbers: 87.18.Mp, 87.18.Cf, 87.18.Tt

Cells continually have to respond to a myriad of signals. One strategy for transmitting distinct stimuli is to use distinct signal transduction networks. It is, however, increasingly recognized that components are often shared between pathways [1]. Moreover, cells can transmit different signals through one and the same pathway, and yet respond to them specifically. In rat cells, for instance, neuronal growth factor and epidermal growth factor stimuli are transmitted through the same MAPK (mitogenactivated protein kinase) pathway, yet give rise to different cell fates, differentiation and proliferation, respectively [2]. These observations suggest that cells are able to transmit multiple messages through the same signal transduction network, just as many telephone calls can be transmitted via a single wire. Indeed, the intriguing question arises whether biochemical networks, like electronic circuits, can multiplex signals: can multiple input signals be combined (encoded) simultaneously in the dynamics of a common signaling pathway, and then decoded such that cells can respond specifically to each signal (see Fig. 1)?

An open question in biology is how cells transduce multiple signals via pathways that share components, since sharing components may lead to unwanted cross talk between the different signals. In recent years, several mechanisms for ensuring signaling specificity have been proposed. One is spatial insulation, where the shared components are incorporated into distinct macromolecular complexes on scaffold proteins $[1,3]$, leading effectively to independent communication channels for the transmission of the respective signals. Other proposals are based on the temporal dynamics of the system, such as crosspathway inhibition [4] and kinetic insulation [5]. With these mechanisms the system cannot be decomposed into independent pathways for the transmission of the

Published by the American Physical Society under the terms of the Creative Commons Attribution 3.0 License. Further distribution of this work must maintain attribution to the author(s) and the published article's title, journal citation, and DOI. respective signals. Yet, these studies suggest that multiple messages cannot be transmitted simultaneously because one pathway tends to dominate the response. Here we demonstrate that cells can truly multiplex signals: we show that they can transmit at least two signals simultaneously through a common pathway, and yet respond specifically to each of them.

Cells employ a number of coding strategies for transducing signals, such as encoding stimuli in the temporal dynamics, like the duration [2] or frequency [6], of an intracellular signal. In principle, any coding strategy could be used to multiplex signals. Here, we consider what is arguably the simplest and most generic coding strategy cells could choose, namely, one in which the signals are encoded in the concentrations of the signaling proteins. We will call this strategy AM multiplexing. (a)

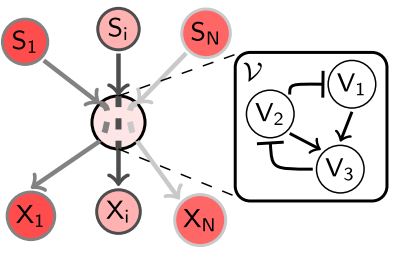

(c)

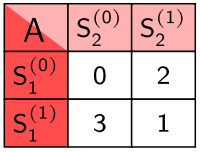

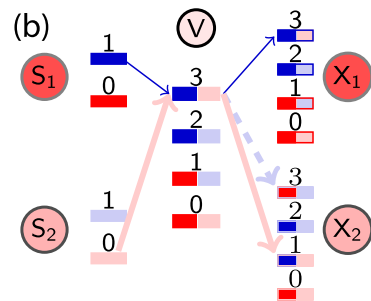

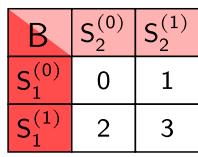

\begin{tabular}{|c|c|c|}
\hline$C$ & $S_{2}^{(0)}$ & $S_{2}^{(1)}$ \\
\hline$S_{1}^{(0)}$ & 0 & 1 \\
\hline$S_{1}^{(1)}$ & 3 & 2 \\
\hline
\end{tabular}

FIG. 1 (color online). (a) Biochemical multiplexing: $N$ different signals are encoded in a common pathway $\mathcal{V}$, and then decoded such that each output species $\mathcal{X}_{i}$ responds to the corresponding input $\mathcal{S}_{i}$. (b) Multiplexing is a mapping problem. The states of two inputs $\mathcal{S}_{1}$ and $\mathcal{S}_{2}$ are mapped onto the concentration of $\mathcal{V}$, which is then mapped onto states of the output species $\chi_{1}$ and $\chi_{2}$; we require that the two lowest (highest) levels of $X_{i}$ correspond to the lowest (highest) level of $S_{i}$. The dashed arrow denotes a mapping that violates this requirement; levels of $V$ and $X_{i}$ are colored according to input pattern $\mathbf{s}=\left(s_{1}, s_{2}\right)$. (c) The 3 distinct mappings of $\mathbf{s}$ to $v$; in panel (b) mapping $C$ is shown. 
We will consider the biochemical network shown in Fig. 1(a). It consists of $N$ input species $\mathcal{S}_{1}, \ldots, \mathcal{S}_{N}$ with copy numbers $S_{1}, \ldots, S_{N}$, a common signal transduction pathway $\mathcal{V}$, and $N$ output species $\chi_{1}, \ldots, \chi_{N}$. The copy number of each input species $S_{i}$ can be in one of $K$ states, $s_{i}=0, \ldots, K-1$, which are labeled in order of increasing copy number, $S_{i}^{(0)}<S_{i}^{(1)}<\cdots<S_{i}^{(K-1)}$. The input pattern is denoted by the vector $\mathbf{s}=\left(s_{1}, \ldots, s_{N}\right)$. Similarly, the copy number of each output species $\mathcal{X}_{i}$ can be in one of $L$ states $X_{i}^{\left(x_{i}\right)}$, with $x_{i}=0, \ldots, L-1$, ordered by increasing copy number, and the output pattern is denoted by the vector $\mathbf{x}=\left(x_{0}, \ldots, x_{N}\right)$. A necessary condition for multiplexing is that the state space of $\mathcal{V}$ is large enough that it is possible to encode the total number of input patterns, $K^{N}$, in $\mathcal{V}$.

We imagine that the $N$ input signals are independent, and that the signal transduction network $\mathcal{V}$ replaces $N$ independent signaling pathways. We therefore require that $X_{i}$ should provide reliable information about the state $s_{i}$, but not necessarily about $s_{j \neq i}$; the $N$ different input signals $\mathbf{s}$ simply have to be transduced to $\mathbf{x}$, not necessarily integrated. In general, however, the state $x_{i}$ will be a function of the states of all the input species: $x_{i}=f(\mathbf{s})$. This reflects the fact that inevitably there is cross talk between the different signals because they are transmitted via the same pathway. However, this cross talk is not detrimental as long as it does not compromise the cell's ability to infer from $x_{i}$ what $s_{i}$ was.

Another key point is that, while the precise mapping from $\mathbf{s}$ to $\mathbf{x}$ may not be critical for the amount of information transmitted per se, this is likely to be important for whether or not this information can be exploited. Let us imagine that the system contains three input species, say, three sugars, each of which is either present or absent, $s_{i}=0$ or 1 ; let us further assume that $\mathcal{X}_{1}$ is an enzyme needed to consume sugar $\mathcal{S}_{i}$. With 8 input patterns $X_{i}$ can, in the absence of noise, take 8 values, identified as states $x_{i}=0, \ldots, 7$. Now, it seems natural to demand that when the sugar $\mathcal{S}_{i}$ is absent $\left(s_{i}=0\right)$, the copy number of enzyme $\mathcal{X}_{i}$ is low, while when $\mathcal{S}_{i}$ is present, the copy number of $\chi_{i}$ is high; this means that the four lowest levels of $X_{i}\left(x_{i}=0,1,2,3\right)$ should correspond to $s_{i}=0$, while the four highest levels of $X_{i}$ should correspond to $s_{i}=1$. We therefore require that the mapping from $\mathbf{s}$ to $\mathbf{x}$ is such that the output states $\left\{x_{i}\right\}$ corresponding to input $s_{i}=j$ are grouped into sets that are contiguous and either increase or decrease monotonically with $j$, for each signal $i$. This leads to a monotonic input-output relation between $S_{i}$ and $X_{i}$ for each $i$. We call this requirement the multiplexing requirement.

In the rest of the Letter, we make these ideas concrete for a network with two input species, $\mathcal{S}_{1}$ and $\mathcal{S}_{2}$, each of which has either a low $\left(s_{i}=0\right)$ or a high concentration $\left(s_{i}=1\right)$, and the shared pathway consists of only one species, $\mathcal{V}$. Multiplexing requires that, in the absence of noise, the four input patterns $\mathbf{S}$ can be mapped onto four distinct states of $V, V^{(v)}$ with $v=0, \ldots, 3$, again labeled in order of increasing copy number. These four levels of $V$ lead to four states for each of the two output species $\chi_{1}$ and $\chi_{2}$ [Fig. 1(b)]. As explained above, we require that we can group these four states into two sets, called low and high, such that the low set, containing $x_{i}=0,1$, corresponds to $s_{i}=0$ and the high set, containing $x_{i}=2,3$, corresponds to $s_{i}=1$ (or vice versa, leading to an inverse input-output relation). We note that there exist different ways of mapping $\mathbf{s}$ to $v$, but not all of these mappings can necessarily be decoded into $\mathbf{x}$ in a manner that satisfies the multiplexing requirement. We therefore first address the question of which combinations of mapping from $\mathbf{s}$ to $v$ and decoding from $v$ to $\mathbf{x}$ fulfill the multiplexing requirement, and then we will discuss what encoding mechanisms actually allow for the required mapping from $\mathbf{s}$ to $v$.

Because of the symmetry in the problem, there are three distinct ways of mapping the four input patterns $\mathbf{s}$ to $v$ [Fig. 1(c)]. To determine whether there exists a scheme for decoding the signals from $v$ to $\mathbf{x}$ that satisfies the multiplexing requirement, we examine for each mapping all possible network topologies between $\mathcal{V}, \chi_{1}$, and $\chi_{2}$, except those that involve autoregulation or mutual regulation since these may lead to bistability. In particular, we allow for activation and repression of $\chi_{1}$ and $\chi_{2}$ by $\mathcal{V}$, and for activation and repression of $\mathcal{X}_{2}$ by $\chi_{1}$, leading to feedforward loops, a common motif in signal transduction pathways and gene networks [7]. In the deterministic mean-field limit the steady-state values of $X_{1}$ and $X_{2}$ are thus given by

$$
\begin{gathered}
X_{1}=k_{1} f\left(V ; K_{\alpha}, n_{\alpha}\right) / \mu, \\
X_{2}=k_{2} f\left(V ; K_{\beta}, n_{\beta}\right) \times f\left(X_{1} ; K_{\gamma}, n_{\gamma}\right) / \mu,
\end{gathered}
$$

where $k$ is the maximum activation or production rate, $\mu$ is the degradation or deactivation rate, and each regulation function is either an activating or repressing Hill function, $f(V ; K, n)=V^{n} /\left(V^{n}+K^{n}\right)$ or $f(V ; K, n)=K^{n} /\left(V^{n}+\right.$ $\left.K^{n}\right)$. The multiplication in Eq. (2) indicates that we assume that at $X_{2}, X_{1}$ and $V$ are integrated according to AND logic [7]. To explore which architectures allow for multiplexing, we performed extensive sampling of the space of parameters $k_{1}, k_{2}, K_{\alpha}, n_{\alpha}, K_{\beta}, n_{\beta}, K_{\gamma}, n_{\gamma}$ for each of the mappings in Fig. 1(c).

Only for mapping $C$ do we find decoding schemes that satisfy the multiplexing requirement for realistic parameter values. Interestingly, all valid decoding networks are incoherent feedforward loops [7]. Figure 2 illustrates the principle for one such motif. $X_{1}(V)$ is a simple activation curve with activation threshold $K_{\alpha}$. In contrast, $X_{2}(V)$ starts low and rises around $K_{\beta}$, but then decreases again due to repression by $\mathcal{X}_{1}$. This nonmonotonicity, which is a result of the incoherent character of the feedforward loop, is critical since this makes it possible to swap the order of the states corresponding to $\mathbf{s}=(1,1)$ and $(1,0)$ in the mapping from $v$ to $x_{2}$. For sharp regulation functions, this yields the intuitive requirement that $V^{(0)}<K_{\beta}<V^{(1)}$ and $X_{1}^{(2)}<K_{\gamma}<X_{1}^{(3)}$. We stress, however, that $n_{\beta}$ and $n_{\gamma}$ do not 

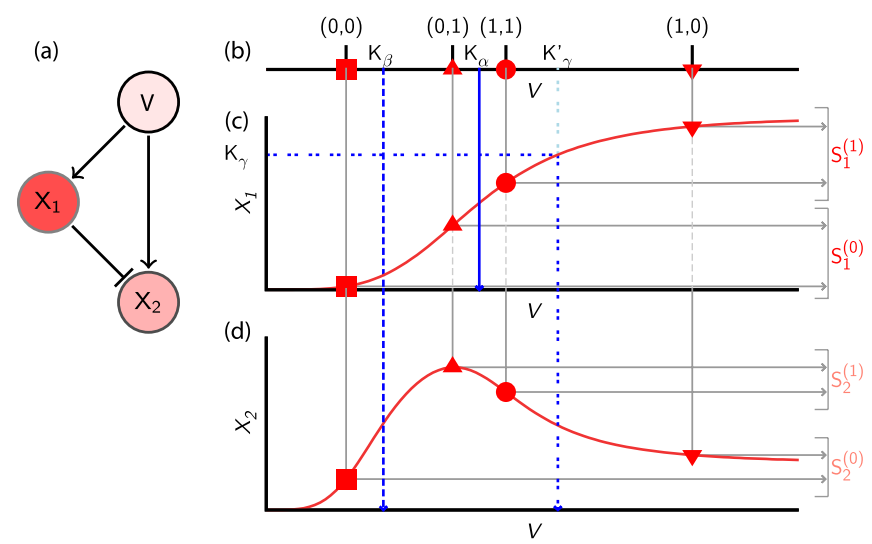

FIG. 2 (color online). Decoding $V$ using an incoherent feedforward loop. (a) Network architecture. (b) The values of $V$ corresponding to the four input patterns $\mathbf{s}$ for mapping $C$ [see Fig. 1(c)], with thresholds $K_{\alpha}, K_{\beta}, K_{\gamma}$ [see Eqs. (1) and (2)]. (c) $X_{1}(V)$. (d) $X_{2}(V)$. The nonmonotonicity of $X_{2}(V)$ swaps the states corresponding to $(1,1)$ and $(1,0)$ in the mapping from $v$ to $x_{2}$.

have to be large (they can be as small as unity), and then $K_{\beta}$ and $K_{\gamma}$ can even fall outside these ranges. In order for $X_{1}$ to be able to repress $X_{2}$ at $X_{1}^{(3)}$ but not $X_{1}^{(2)}, X_{1}$ should not be saturated at $V^{(2)}$; this means that $K_{\alpha} \gtrsim V^{(2)}$ and $n_{\alpha}$ should not become so large that $X_{1}(V)$ becomes two-valued. These mild constraints indicate that this is a robust decoding scheme that can be implemented for a wide range of parameter combinations.

We can now also understand why mappings $A$ and $B$ are difficult to decode: they would require an input-output relation between $X_{2}$ and $V$ that rises more than once. This is difficult to achieve in a feedforward loop without mutual repression or activation.

The above analysis shows that it is possible to decode multiple signals simultaneously, provided that the input $\mathbf{s}$ can be encoded in $V$ according to mapping $C$. The next question is how this mapping, which corresponds to particular input-output relation $V\left(S_{1}, S_{2}\right)$, can be generated. Multiplexing is most beneficial when the input signals are multiplexed at the beginning of signal transmission. We therefore consider whether two input signals can be multiplexed at the level of a single protein $\mathcal{V}$, which could be an enzyme or a receptor at the very beginning of a signaling cascade. We consider a canonical motif where $\mathcal{S}_{1}$ and $\mathcal{S}_{2}$ bind competitively to $\mathcal{V}$, which can exist in either an active conformational state $A$ or an inactive state $I$ [Fig. 3(a)]. In equilibrium the mean number of active $\mathcal{V}$ molecules will be

$$
V\left(S_{1}, S_{2}\right)=\frac{V^{\max }\left(1+q_{1}^{A}+q_{2}^{A}\right)}{1+q_{1}^{A}+q_{2}^{A}+e^{\Delta E_{0}}\left(1+q_{1}^{I}+q_{2}^{I}\right)},
$$

where $V^{\max }$ is the total number of $\mathcal{V}$ molecules, $\Delta E_{0}=$ $E_{0}^{A}-E_{0}^{I}$ is the free-energy difference between the active and inactive states of $V$ in the absence of ligand binding, and $q_{i}^{I, A}=S_{i} / K_{i}^{I, A}$ with $K_{i}^{I}$ and $K_{i}^{A}$ the dissociation constants for the binding of $\mathcal{S}_{i}$ to inactive and active $\mathcal{V}$,
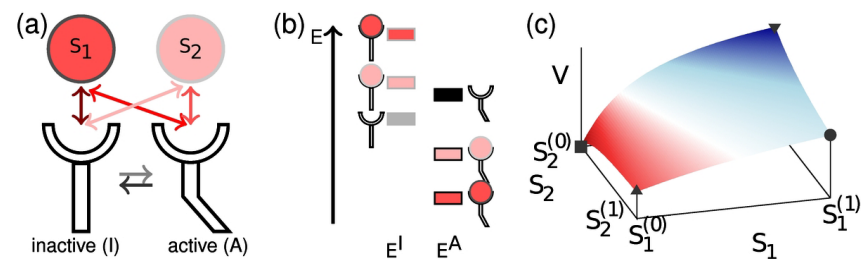

FIG. 3 (color online). (a) An encoding scheme where two ligands $\mathcal{S}_{1}$ and $\mathcal{S}_{2}$ competitively bind to a protein $\mathcal{V}$, which can be in an active state $A$ or an inactive state $I$. (b) Energy levels of $\mathcal{V}$ in the active or inactive state with no ligand, ligand $\mathcal{S}_{1}$, or ligand $\mathcal{S}_{2}$ bound; $e^{-E_{i}^{I}} \equiv S_{i} / K_{i}^{I} ; e^{-E_{i}^{A}} \equiv e^{-\Delta E_{0}} S_{i} / K_{i}^{A}$. (c) Inputoutput relation $V\left(S_{1}, S_{2}\right)$ corresponding to the energy levels in (b), yielding mapping $C$ [Fig. 1(c)]; symbols $\mathbf{\square}, \boldsymbol{\Lambda}, \boldsymbol{O}, \boldsymbol{\nabla}$ correspond to states in Fig. 2.

respectively. We find that a scheme where $\mathcal{S}_{1}$ activates $\mathcal{V}$ more strongly than $\mathcal{S}_{2}$, giving energy levels as in Fig. 3(b), generates the required $V\left(S_{1}, S_{2}\right)$ [Fig. 3(c)]; competition between the two ligands reduces the probability of $S_{1}$ binding the receptor when $S_{2}$ is present, ensuring that $V\left(S_{1}^{(0)}, S_{2}^{(1)}\right)<V\left(S_{1}^{(1)}, S_{2}^{(1)}\right)<V\left(S_{1}^{(1)}, S_{2}^{(0)}\right)$. In the limits $S_{i}^{(0)} \ll K_{i}^{I, A} \ll S_{i}^{(1)}$ a sufficient condition for this ordering is $1>K_{2}^{A} / K_{2}^{I}>K_{1}^{A} / K_{1}^{I}$. Clearly, therefore, the required encoding $V\left(S_{1}, S_{2}\right)$ could be implemented at the level of a single signaling protein $\mathcal{V}$.

The analysis above shows that in principle biochemical networks can multiplex signals in the mean-field, deterministic limit. However, there remains the question of whether signals can be multiplexed reliably in the presence of inevitable biochemical noise. To address this, we estimate a lower bound on the information about two binary signals $\mathcal{S}_{1}$ and $\mathcal{S}_{2}$ that are transmitted through the network studied above [Eqs. (1)-(3)]. We define the total information $I \equiv I\left(S_{1}, X_{1}\right)+I\left(S_{2}, X_{2}\right)$ as the sum of the mutual information for each of the individual signals [8]. Note that in the presence of noise $X_{i}$ is not limited to 4 states but can in principle take any value. This definition of $I$ makes it straightforward to directly compare the performance of this network with that of two independent pathways. If each of the two input states for each $S_{i}$ is equally likely, then the maximum value of $I\left(S_{i}, X_{i}\right)$ is 1 bit for each signal $i$; the maximum value of $I$ is thus 2 bits.

To maximize the lower bound on $I$ we optimize the network parameters using a simulated-annealing algorithm. We fix the deactivation rate of $\chi_{1}$ and $\chi_{2}$ to be $\mu=1 \mathrm{~s}^{-1}$ and set $n=n_{\alpha}=n_{\beta}=n_{\gamma}=2$; the results are insensitive to the precise value of $n$ for $1 \leq n \leq 4$. We set the maximum value of each $X_{i}$ to be $X^{\max }$; this specifies the activation rates $k_{1}$ and $k_{2}$. We optimize the remaining decoding parameters $K_{\alpha}, K_{\beta}$, and $K_{\gamma}$ over the range $\left[0, V^{\max }\right]$ or $\left[0, X^{\max }\right]$ as appropriate, and the encoding parameters $q_{i}^{j}$ and $\Delta E$ over the range $\left[10^{-3}, 10^{3}\right]$ and $\left[-10 k_{B} T, 10 k_{B} T\right]$, respectively. Ligand binding is assumed to be fast compared to receptor activity switching; the rate of switching of $\mathcal{V}$ from the active to the inactive state is fixed at $1 \mathrm{~s}^{-1}$, and the switching rate in the reverse 


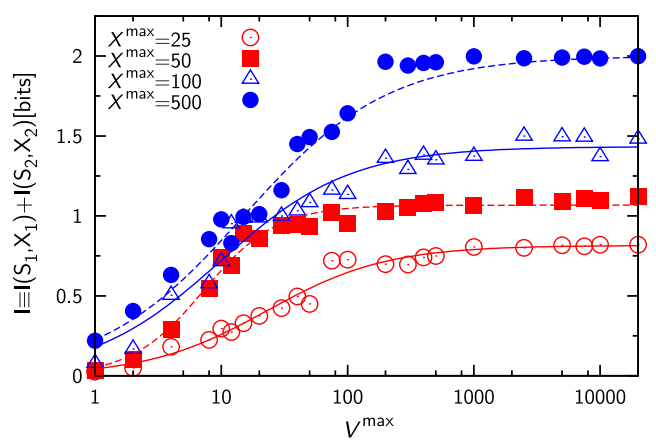

FIG. 4 (color online). The transmitted information $I \equiv$ $I\left(S_{1}, X_{1}\right)+I\left(S_{2}, X_{2}\right)$ as a function of $V^{\max }$ for four different values of $X^{\mathrm{max}}$. I can reach the maximum value of 2 bits provided $V^{\max }$ and $X^{\max }$ are large enough. Typical values for the optimized parameters are $K_{\alpha} \sim V^{\max }, K_{\beta} \sim 0.3 V^{\max }, K_{\gamma} \sim$ $0.5 X^{\max }, \Delta E_{0} \approx-2 k_{B} T, S_{1}^{(0)}=S_{2}^{(0)}=0, K_{1}^{I} \gg S_{1}^{(1)}, K_{2}^{I} \sim S_{2}^{(1)}$, $K_{1}^{A} \ll S_{1}^{(1)}, K_{2}^{A}<S_{2}^{(1)}$.

direction then follows from detailed balance given Eq. (3). For each parameter set we compute the mutual information using the linear-noise approximation [8,9]. Its accuracy was verified by performing Gillespie simulations of the optimized networks [10].

Using this procedure we compute $I$ as a function of $V^{\max }$ and $X^{\max }$, which determine the intrinsic noise in the encoding and decoding processes. Figure 4 shows that below a threshold copy number $V_{c}^{\max } \approx 10$ the total information is low regardless of $X^{\text {max }}$ because four distinct states of $V$ cannot be generated. Once $V^{\max }$ becomes sufficiently large that the four encoded signals are well separated, $I$ saturates at a value determined by the level of noise in the production and decay of $X_{i}$. For large $X^{\max }$ the information $I$ reaches 2 bits, thus matching the performance of two independent channels. Importantly, $I$ reaches 2 bits for $V^{\max } \approx X^{\max } \approx$ 500 , which is well within the range of typical protein copy numbers inside living cells. This shows that biochemical networks can multiplex two signals reliably at biologically relevant noise levels.

In summary, our results suggest that cells can transmit at least two binary signals through one and the same pathway, and yet respond specifically and reliably to each of them. The proposed mechanism for biochemical multiplexing is based on swapping the order of states during the encoding and decoding steps. It is clear that the principle is generic, and could be implemented in any biochemical network that uses an incoherent feedforward loop [7]. Indeed, the architecture of a number of well-studied systems resembles that of the system studied here. It is well known that G-protein coupled receptors (GPCRs) can be stimulated by many different ligands, yet give rise to different cell fates, a phenomenon referred to as "multiplicity" [11]. A GPCR activates two $\mathrm{G}$ proteins, $\mathrm{G} \alpha$ and $\mathrm{G} \beta \gamma$. Of particular interest is the system where $\mathrm{G} \alpha_{q}$ activates RhoGef63 [12], leading to cytoskeletal remodeling, while $\mathrm{G} \beta \gamma$ activates $\operatorname{PLC} \beta$ [13], ultimately controlling cell proliferation. Interestingly, PLC $\beta$ binds not only $\mathrm{G} \beta \gamma$, but also $\mathrm{G} \alpha_{q}$, thereby inhibiting the activation of RhoGef by $\mathrm{G} \alpha$ [12]. Hence, we have a scheme where one protein $\mathcal{V}$ (the GPCR) activates two proteins $\chi_{1}$ (RhoGef63) and $\chi_{2}$ $(\operatorname{PLC} \beta)$, whereby $\chi_{2}$ effectively inhibits $\chi_{1}$. This is highly similar to our proposed multiplexing scheme, and it is indeed tempting to speculate that multiplicity is achieved via multiplexing. Secondly, Ras is a major hub in cell signaling: it is activated by many cellular stimuli, including growth factors, differentiation factors and cell survival factors. Activation of Ras leads to the activation of the Raf-Mek-Erk MAPK pathway, ultimately leading to differentiation or proliferation, and also via PI3K to the activation of Akt and hence the mTOR pathway, controlling many processes such as metabolism. Importantly, Akt also deactivates $\operatorname{Raf}[14]$, generating a $\operatorname{Ras}(\mathcal{V})$-Raf $\left(\chi_{1}\right)$ Akt $\left(\chi_{2}\right)$ incoherent feedforward loop. Finally, CREB, a major transcription factor in neuronal cells, is activated by many different stimuli, and, in turn, regulates many different target genes, some of which include incoherent feedforward loops [15]. It will be interesting to test whether these systems employ biochemical multiplexing when they are stimulated by two signals simultaneously.

We thank Tom Shimizu, Frank Bruggeman, and Nils Blüthgen for a critical reading of the manuscript. This work is supported by FOM/NWO.

[1] M. A. Schwartz and H. D. Madhani, Annu. Rev. Genet. 38, 725 (2004).

[2] C. J. Marshall, Cell 80, 179 (1995).

[3] J.C. Patterson, E.S. Klimenko, and J. Thorner, Sci. Signal. (Online) 3, ra75 (2010).

[4] L. Bardwell, X. Zou, Q. Nie, and N. L. Komarova, Biophys. J. 92, 3425 (2007); M. N. McClean et al., Nat. Genet. 39, 409 (2007); B. Hu, W.-J. Rappel, and H. Levine, Biophys. J. 96, 4755 (2009); L. Rensing and P. Ruoff, J. Theor. Biol. 257, 578 (2009).

[5] M. Behar, H. G. Dohlman, and T. C. Elston, Proc. Natl. Acad. Sci. U.S.A. 104, 16146 (2007).

[6] M.J. Berridge, P. Lipp, and M. D. Bootman, Nat. Rev. Mol. Cell Biol. 1, 11 (2000).

[7] S. S. Shen-Orr et al., Nat. Genet. 31, 64 (2002).

[8] C. E. Shannon, Bell Syst. Tech. J. 27, 379 (1948); E. Ziv et al., PLoS ONE 2, e1077 (2007); P. Mehta et al., Mol. Syst. Biol. 5, 325 (2009); A. M. Walczak, G. Tkačik, and W. Bialek, Phys. Rev. E 81, 041905 (2010).

[9] N. G. van Kampen, Stochastic Processes in Physics and Chemistry (Elsevier, Amsterdam, 2001).

[10] D. T. Gillespie, J. Phys. Chem. 81, 2340 (1977).

[11] E. Hermans, Pharmacol. Therapeut. 99, 25 (2003).

[12] S. Lutz et al., J. Biol. Chem. 280, 11134 (2005); G. L. Waldo et al., Science 330, 974 (2010); F. Philip et al., Curr. Biol. 20, 1327 (2010).

[13] S. G. Rhee and Y.S. Bae, J. Biol. Chem. 272, 15045 (1997).

[14] S. Zimmermann and K. Moelling, Science 286, 1741 (1999).

[15] H. D. MacGillavry et al., J. Neurosci. 29, 15542 (2009). 